\title{
Médiévales
}

Langues, Textes, Histoire

72 | printemps 2017

Roman du Genji et société aristocratique au Japon

\section{Le Roman du Genji et la société aristocratique du Japon ancien}

The Tale of Genji and Aristocratic Society in Ancient Japan

Daniel Struve et Terada Sumie

\section{(2) OpenEdition}

1 Journals

\section{Édition électronique}

URL : https://journals.openedition.org/medievales/8021

DOI : 10.4000/medievales.8021

ISSN : $1777-5892$

Éditeur

Presses universitaires de Vincennes

Édition imprimée

Date de publication : 22 juin 2017

Pagination : $5-20$

ISBN : 978-2-84292-612-0

ISSN : 0751-2708

\section{Référence électronique}

Daniel Struve et Terada Sumie, "Le Roman du Genji et la société aristocratique du Japon ancien », Médiévales [En ligne], 72 I printemps 2017, mis en ligne le 28 février 2019, consulté le 22 avril 2022. URL : http://journals.openedition.org/medievales/8021 ; DOI : https://doi.org/10.4000/medievales. 8021 
Daniel Struve

Terada Sumie

\section{Le Roman du Genji et la société aristocratique du Japon ancien}

$\mathrm{Au}$ début du XI siècle, à l'heure où Murasaki Shikibu prenait le pinceau pour rédiger le Roman $d u$ Genji, le Japon était soumis à l'autorité d'une cour impériale dominée par quelques familles aristocratiques. Selon certaines estimations, l'archipel aurait compté alors une population de sept à huit millions d'habitants ${ }^{1}$, dont 100000 environ étaient rassemblés dans la capitale du pays, Heian-kyô, l'actuelle ville de Kyôto (fig. 1). Parmi eux, un millier environ constituait la noblesse de cour (du $1^{\text {er }}$ au $5^{\mathrm{e}} \mathrm{rang}$ ), familles comprises, dont la plus haute sphère (du $1^{\mathrm{er}}$ au $3^{\mathrm{e}}$ rang) ne comptait guère qu'une centaine de personnes ${ }^{2}$. Une culture d'un très grand raffinement avait pu naître et s'épanouir en assimilant les apports du continent venus de la grande civilisation de la Chine des Tang, dans un contexte où pouvoir et richesse étaient concentrés dans les mains d'une poignée d'hommes placés à la tête d'une organisation administrative centralisée couvrant l'ensemble du territoire. Nous nous proposons, dans cette introduction, de présenter un aperçu de cette société en nous limitant aux éléments indispensables pour situer et comprendre le Roman du Genji.

1. F. HÉrAIL, La Cour du Japon à l'époque de Heian, aux Xe et XI siècles, Paris, 1995, p. 9.

2. H. OBoroya, «Nihon kodai no kizoku » (La noblesse de l'époque ancienne du Japon), dans Kuge to buke sono hikaku bunmeishiteki kenkyû (Étude comparee de l'histoire culturelle des nobles et des guerriers), Actes des colloques organisés par le Centre international de recherches pour les études japonaises (Nichibunken), vol. 22, Kyôto, 2004, p. 189-196. 


\section{Le cadre historique}

C'est au VII ${ }^{\mathrm{e}}$ siècle de l'ère chrétienne qu'à la suite de l'introduction du bouddhisme et de l'écriture chinoise, le Japon adopta un système de gouvernement imité de la Chine impériale, ce qui lui permettait d'entrer de plain-pied dans la culture continentale. Sur le modèle chinois, un code administratif et un code pénal furent rédigés à la fin du VII ${ }^{\mathrm{e}}$ et au début du VIII ${ }^{\mathrm{e}}$ siècle, fixant les moindres aspects du gouvernement et de l'administration de la cour et du pays. L'État fondé sur les codes institués au début du VIII ${ }^{\mathrm{e}}$ siècle se maintient tout au long de l'époque de Nara (VIII ${ }^{\mathrm{e}}$ siècle), puis de celle de Heian ( $\mathrm{IX}^{\mathrm{e}}-\mathrm{XII}^{\mathrm{e}}$ siècles), même si une partie des codes cesse d'être appliquée à partir du $\mathrm{X}^{\mathrm{e}}$ siècle. Autour de l'empereur, une aristocratie de cour domine le pays. En 794, la capitale est transférée de Nara à Heian-kyô, l'actuelle Kyôto, située dans la partie centrale du Japon au croisement des principales voies de communication. Une grande partie des richesses des provinces est acheminée dans cette capitale, favorisant l'essor d'une brillante culture.

Une famille occupe dans ce système une place prépondérante : celle des Fujiwara, descendants de Nakatomi no Kamatari (614-659) et de son fils Fuhito (659-720), qui ont joué un rôle déterminant dans l'élaboration des codes. À partir du milieu du IX ${ }^{\mathrm{e}}$ siècle, la branche dite du Nord de cette famille prend le contrôle de la cour grâce à une politique d'alliance matrimoniale avec la famille impériale qui permet aux dignitaires Fujiwara de faire monter sur le trône leurs propres petits-fils, nés de leurs filles, dont ils deviennent les régents. Le pouvoir des Fujiwara se maintiendra jusqu'en 1086, tant que les régents auront des filles qu'ils pourront donner pour épouses aux empereurs. Maîtres des promotions grâce au droit de regard qu'ils obtiennent sur toutes les décisions impériales, les régents Fujiwara accroissent leurs domaines et nouent des liens de clientèle avec les gouverneurs de province qui assurent leur prospérité. La figure de Fujiwara no Michinaga (966-1027), le plus brillant et le plus puissant des régents Fujiwara, dont il est question dans l'article de Charlotte von Verschuer qui ouvre ce dossier, marque l'apogée de la civilisation de cour de l'époque de Heian. C'est dans son entourage que naît le chef-d'œuvre reconnu de la littérature japonaise, le Roman du Genji.

En même temps que les régents Fujiwara s'imposaient en étroite liaison avec la famille impériale comme le pouvoir dominant à la cour, les provinces connaissaient aussi une évolution qui, à terme, allait miner l'emprise de la cour sur le pays. Le système de redistribution des rizières, adopté conformément aux codes, est progressivement abandonné et devient obsolète à partir du début du $\mathrm{X}^{\mathrm{e}}$ siècle. Les gouverneurs de province, issus des familles de fonctionnaires de rang moyen, s'appuient sur les notables locaux et le personnel qu'ils emmènent avec eux depuis la capitale. Ils 


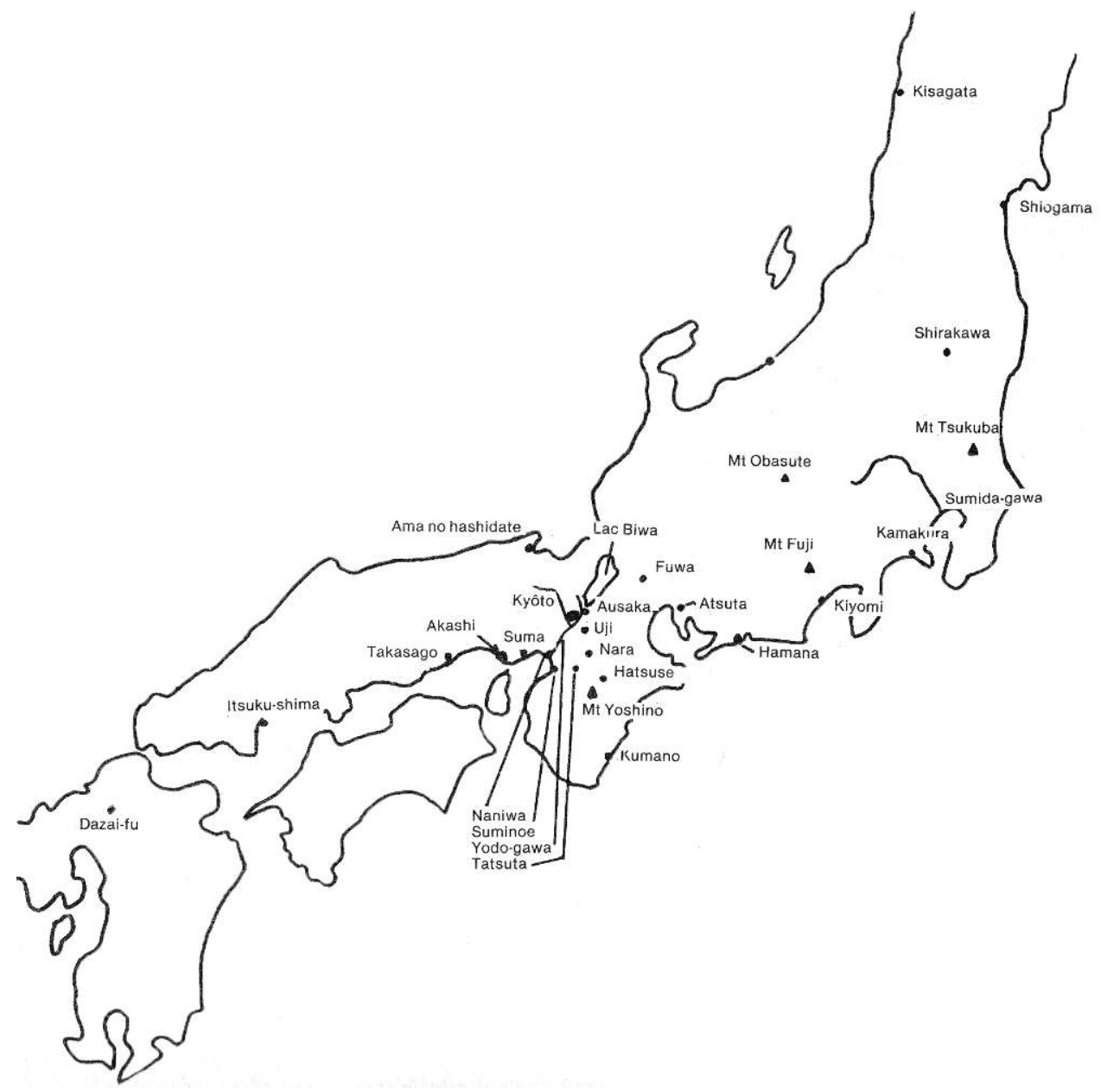

Fig. 1. Carte du Japon indiquant les principaux sites chantés dans les waka ainsi que les centres administratifs

Carte extraite de J. Pigeot, Michiyuki-bun. Poétique de l'itinéraire dans la littérature du Japon

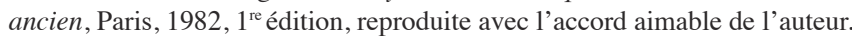


gèrent les provinces d'une manière de plus en plus autonome, à charge pour eux d'acheminer à la cour les redevances attendues. La cour jouit d'un prestige qui lui permet de rester source de légitimité et arbitre des conflits. Cependant, le rapport des forces évolue en faveur des provinces. Une noblesse locale, faite de descendants de familles aristocratiques n'ayant pas trouvé d'emploi à la capitale ou de notables locaux, s'affirme et se militarise afin d'assurer un ordre que la cour ne peut plus garantir à elle seule ${ }^{3}$.

Cette déliquescence de l'État fondé sur les codes prend une tournure dramatique au milieu du $\mathrm{XII}^{\mathrm{e}}$ siècle quand les guerriers se trouvent en position d'arbitrer les conflits à la cour. Ce tournant marque le début de l'âge des guerriers et de la longue période allant jusqu'au milieu du XVI ${ }^{\mathrm{e}}$ siècle, désignée au Japon comme le « Moyen Âge » (chûsei). La période allant du $\mathrm{VII}^{\mathrm{e}}$ au XII ${ }^{\mathrm{e}}$ siècle avec la gestation, puis la mise en place de l'État fondé sur les codes (époques d'Asuka et de Nara), puis l'établissement de la capitale à Heian-kyô et la montée en puissance de la famille Fujiwara, remplacée à partir de 1086 par la maison des Empereurs retirés, est, elle, désignée comme l' «Âge ancien » ou l' «Antiquité » (kodai $)^{4}$. On observe donc un décalage entre la périodisation japonaise et la périodisation occidentale. Ensemble, l' « Âge ancien » (VII $-\mathrm{XII}^{\mathrm{e}}$ siècles) et le « Moyen Âge » japonais correspondent à peu près à la période médiévale occidentale. En littérature, l'époque d'Asuka et de Nara (VII et VIII ${ }^{\mathrm{e}}$ siècles) est connue comme l'époque antique (jôdai). L'époque de Heian correspond, elle, à l'âge classique et connaît, outre la floraison des lettres chinoises, l'émergence d'une riche littérature en langue vernaculaire, au sein de laquelle un rôle éminent est joué par les femmes.

\section{Les grands traits de la culture de l'époque de Heian}

La généralisation progressive de l'écriture et de la culture continentale, au cours du VII ${ }^{\mathrm{e}}$ siècle, se traduit par la formation d'une culture de cour qui rayonne dans l'ensemble du pays grâce à l'établissement de capitales provinciales et d'un réseau d'institutions monastiques. La

3. Cf. F. HÉRAIL, Gouverneurs de province et guerriers dans les Histoires qui sont maintenant du passé, Paris, 2004. Cette sélection d'anecdotes du célèbre recueil Les Histoires qui sont maintenant du passé (Konjaku monogatari shû) (XII ${ }^{\mathrm{e}}$ siècle) illustre d'une manière très vivante l'émergence d'une culture guerrière à partir du $\mathrm{X}^{\mathrm{e}}$ siècle.

4. Aujourd'hui cependant les historiens ont tendance à avancer la limite inférieure du Moyen Âge japonais au début du XII ${ }^{\mathrm{e}}$ siècle pour mieux tenir compte des transformations économiques et sociales qui interviennent à la fin de l'époque de Heian, notamment le développement du système des domaines supportant une multitude de droits depuis ceux des paysans cultivateurs jusqu'à ceux des grandes maisons aristocratiques ou institutions religieuses. Voir F. HÉRAIL et al. éd., Histoire du Japon, Lyon, 1990, p. 118 sq. 
poésie en chinois de caractère cérémoniel se développe dans l'entourage de l'empereur Tenji (626-672, qui régna de 668 à 672), à la cour d'Ômi sur le lac Biwa. Elle fleurit aussi dans la première moitié du IX siècle à la cour de l'empereur lettré et calligraphe Saga (786-842, qui régna de 809 à 823 ), et connaît son apogée avec l'œuvre du poète en chinois et homme d'État Sugawara no Michizane, issu d'une vieille famille de lettrés liée à l'Office des Études supérieures ou Université (Daigakuryô) ${ }^{5}$. Les familles de la grande aristocratie, qui n'envoient pas leurs enfants à l'Université, les forment dans leurs propres institutions, comme celle de la famille Fujiwara, dotée d'une riche bibliothèque. Plus encore que la connaissance des classiques du confucianisme, est prisée à la cour l'habileté à composer en poésie et en prose chinoises, ce qui explique le prestige dont jouit entre toutes la Voie des Lettres.

Les autres formes d'expression artistiques ne sont pas moins fleurissantes dans une société où les rites et les cérémonies, publiques ou privées, jouent un rôle prédominant. La vie du palais et de la capitale est rythmée par les différents événements annuels (nenjû gyôji), dont le déroulement est réglé dans les moindres détails et qui font l'objet de minutieuses descriptions dans les notes journalières des courtisans afin de servir de précédents pour la postérité. La musique instrumentale est omniprésente dans les cérémonies et les distractions de la cour ou dans les demeures aristocratiques. La peinture d'inspiration chinoise (karae) ou japonaise (yamatoe) décore les paravents et les portes coulissantes du palais impérial et des résidences aristocratiques. Les diverses fonctions de la peinture sont analysées ici par Estelle Leggeri-Bauer, notamment la dimension de faste cérémoniel que met en valeur le livre « Les Concours de peintures » du Roman du Genji. La poésie en chinois et en japonais donne lieu, elle aussi, à des rassemblements de caractère cérémoniel, comme ceux qu'évoque, dans son étude, Michel Vieillard-Baron.

La prose en chinois joue un rôle essentiel dans le fonctionnement de l'État bureaucratique calqué sur le modèle chinois depuis ses débuts. Les généalogies et les mythes nationaux ou locaux, transmis jusqu'alors par voie orale, ont été compilés et couchés par écrit par Ô no Yasumaro dans les Récits des temps anciens (Kojiki, 708). Avec les Chroniques du Japon (Nihon shoki, 721), suivies par cinq autres histoires réalisées entre 797 et

5. Institution destinée à la formation des futurs fonctionnaires, créée par les codes. Outre la Voie des Lettres, on y enseignait également les classiques confucéens, le droit et les mathématiques. L'Office des Études supérieurs suivit le destin des codes et perdit de son importance à mesure que les nominations furent décidées sur d'autres critères que la réussite aux examens. Il cessa progressivement de fonctionner au cours du XII ${ }^{\mathrm{e}}$ siècle. Voir H. YAMANAKA, K. SUZUKI éd., Heian kizoku no kankyô : Heian jidai no bungaku to seikatsu (Le milieu des nobles de Heian : littérature et vie quotidienne à l'époque de Heian), Tôkyô, 1994, p. 193-207. 
901 , le Japon se dote d'une histoire officielle, à la manière chinoise. Après cette date, la compilation d'historiographies officielles est abandonnée, de même, du reste, que l'envoi d'ambassades en Chine.

Il convient enfin de signaler que la connaissance du chinois comprenait aussi l'étude des écritures bouddhiques, enseignées dans des monastères qui entretenaient des liens étroits avec la cour et l'aristocratie. Les enseignements qui y étaient dispensés ont exercé une profonde influence sur la culture de cour. On peut ainsi citer la figure du moine Kûkai (774-835), introducteur au Japon de l'école ésotérique Shingon, mais aussi auteur d'un premier traité de poétique chinoise. Ou celle, plus tardive, du lettré et fonctionnaire dévot Yoshishige no Yasutane (933-1002), proche du régent Fujiwara no Michinaga, qui fut un des initiateurs des réunions du Kangakukai (Assemblée pour la promotion de l'étude), où moines et laïcs se réunissaient afin de réciter conjointement le Sûtra du Lotus et composer des poèmes en chinois dont l'inspiration bouddhique rejoignait celle du poète chinois Bai Juyi (772-846).

Pensée et croyances bouddhiques imprègnent toute la culture de l'époque de Heian et l'attrait pour la retraite religieuse, fondé sur une prise de conscience de la vanité de la vie dans le monde, est un thème qui traverse de part en part le Roman du Genji. Pratiqué au sein du Tendai, l'amidisme ou croyance en la Terre pure du bodhisattva Amitabha (Amida en japonais), qui a fait le vœu de sauver tous les êtres invoquant son nom, se répandit dans les milieux aristocratiques, notamment sous l'influence de l'ouvrage du moine Genshin (942-1017), le Compendium pour la renaissance dans la Terre pure (Ôjô yôshû, 985) ${ }^{6}$. Le monde était censé entrer prochainement dans la période de la « fin de la Loi » (mappô), dans laquelle la prédication de la Loi apportée par le Bouddha historique était si dégradée que le salut n'y était plus guère possible que par la «force d'autrui », en l'occurrence celle du vœu d'Amida. Fujiwara no Michinaga fait construire pour sa retraite le temple Hôjôji, dédié au culte d'Amida, et y meurt avec l'espérance de renaître dans la Terre pure.

Si nous avons insisté sur l'importance de l'élément chinois, c'est qu'il constitue le cœur de la culture de l'époque classique. Il ne s'agit pas pour autant d'exclure la littérature d'expression japonaise. Dès le VII ${ }^{\mathrm{e}}$ siècle se développe au Japon à côté des lettres chinoises une poésie en langue vernaculaire, le waka (ou yamato-uta, littéralement « chant japonais »). Une partie importante de cette production a été recueillie dans l'Anthologie des Dix mille feuilles (Man.yôshû), dont la dernière compilation date d'environ 780. Dès cette époque, la poésie en japonais, contrairement à la culture chinoise, faisait la part belle aux femmes. Le poète de cour et

6. GENSHIN, The Essentials of Salvation (Ôjô yôshû), trad. partielle P. YAMPOLSKY, dans W. T. DE BARY éd., Sources of Japanese Tradition, vol. 1, New York, 2001. 
fonctionnaire Ki no Tsurayuki (866 ?-945 ?) acheva de donner à la poésie japonaise sa forme classique et assura définitivement sa place parmi les activités officielles de la cour, en participant à la compilation de la première anthologie impériale de poésie japonaise, le Recueil des poèmes anciens et modernes (Kokin wakashû). Il en rédigea également la préface en japonais, posant ainsi le début de la réflexion poétique proprement japonaise. La forme longue (chôka), qui avait fleuri au VII ${ }^{\mathrm{e}}$ et $\mathrm{VIII}^{\mathrm{e}}$ siècle, ne se maintient alors qu'à titre d'archaïsme, laissant toute sa place à la forme courte de 31 syllabes $(\text { tanka })^{7}$, fortement codifiée tant du point de vue du vocabulaire que des thèmes traités : saisons, amour, voyage, deuil, séparation, célébrations. Cette poésie en langue vernaculaire remplit une fonction sociale aussi bien que cérémonielle.

$\mathrm{Au}$ début $\mathrm{du} \mathrm{XI}^{\mathrm{e}}$ siècle, au côté de nombreuses figures féminines, dont beaucoup s'illustrent également en prose, mérite d'être mentionné le nom du poète et poéticien Fujiwara no Kintô (966-1041), auteur de nombreux traités et compilateur du recueil Poèmes japonais et chinois à réciter (Wakan rôeish $\hat{u}$ ), où sont appariés poèmes japonais et fragments de poèmes chinois. Ce recueil, qui devait connaître un immense succès, consacre le double caractère chinois et japonais de la culture de cour, analysé dans l'article d'Ivo Smith. Fukutô Sanae aborde, elle, la part jouée dans la culture féminine par cette double culture poétique.

Malgré sa brièveté, la poésie en japonais (waka), et tout particulièrement la poésie amoureuse, possède également une dimension narrative. Tout poème d'amour - et a fortiori tout échange poétique entre amants - s'inscrit au moins virtuellement dans une histoire, souvent évoquée dans les brèves introductions en prose (kotobagaki) qui précèdent de nombreux poèmes. Cette dimension, déjà présente dans le Man.yôshû, est héritée par les recueils de poésie waka de l'âge classique. Elle est à l'origine de la forme mixte, mélangeant poésie et prose, que sont les récits à poèmes (uta monogatari), dont le plus fameux est le recueil intitulé Contes d'Ise, regroupant de courts récits intégrant un ou plusieurs poèmes. Ce recueil se serait constitué progressivement, sans doute au $\mathrm{X}^{\mathrm{e}}$ siècle, autour des poèmes et de la figure du poète Ariwara no Narihira (825-880), parangon de raffinement et d'élégance et un des modèles du héros du Roman du Genji.

$\mathrm{Au}$ début du $\mathrm{X}^{\mathrm{e}}$ siècle naît le récit de fiction ou monogatari. Tout en incluant de nombreux éléments merveilleux, il est consacré à la peinture

7. Cette forme courte, souvent identifiée au waka, a connu une extraordinaire fortune et se maintint jusqu'au XIX ${ }^{\mathrm{e}}$ siècle. Influençant profondément la culture japonaise, elle fut à l'origine d'autres formes poétiques comme la poésie en chaîne (renga, consistant à enchaîner vers de 17 et de 14 syllabes), qui fleurit au XIV et au XVI ${ }^{\mathrm{e}}$ siècle, ou une version plus populaire de celle-ci, le haikai, dont l'apogée se situe au XVII' siècle. Une forme moderne du tanka, ainsi que le célèbre haiku en 17 syllabes, dérivé du haikai, occupent toujours une place importante dans la littérature contemporaine. 
du milieu aristocratique et fait la part belle aux intrigues politiques et amoureuses. Mais, contrairement à la poésie japonaise, il ne possède aucun caractère officiel. Si une trentaine de titres sont connus, très peu d'œuvres ont été conservées. Outre le Conte du Coupeur de bambou ${ }^{8}$, qui comprend encore de nombreux traits hérités du conte, deux œuvres romanesques de la fin du $\mathrm{X}^{\mathrm{e}}$ siècle nous sont parvenues, Le Roman de l'arbre creux (Utsuho monogatari) et le Roman de la chambre basse (Ochikubo monogatari), qui témoignent du haut degré d'élaboration qu'avait alors acquis le genre romanesque. Le roman est considéré avant tout comme un pur divertissement. Mais, dans la mesure où il intègre la description d'événements de la cour (Roman de l'arbre creux) ou des mœurs domestiques des grandes familles (Roman de l'arbre creux, Roman de la chambre basse) ${ }^{9}$, il acquiert le caractère d'une chronique parallèle, ouvrant sur une réflexion politique et morale.

On ne saurait enfin comprendre l'apparition du roman de cour sans mentionner deux autres développements importants : les Mémoires d'une Éphémère (Kagerô no nikki), autobiographie d'une épouse du régent Fujiwara no Kaneie, connue comme la Mère de Michitsuna (936 ?-995), et les Notes de chevet, rédigées par la dame du palais Sei Shônagon (966 ?-1024 ?). Ces deux œuvres marquent le début de la prédominance des femmes dans la littérature vernaculaire. Dans une société aristocratique où les alliances matrimoniales jouent un rôle décisif pour l'acquisition du pouvoir et des richesses, l'éducation des filles, décrite dans l'article de Fukutô Sanae, devient une nécessité. Dans l'entourage des impératrices ou des grandes dames de l'aristocratie se constituent de brillantes sociétés féminines qu'on a pu qualifier de «salons », où la maîtrise de la poésie japonaise et des arts d'agrément sont indispensables. Les Mémoires d'une Éphémère, dont l'auteur évoque sa liaison de vingt ans avec un des plus hauts personnages du temps, innovent par leur écriture aux longues phrases allusives qui mettent au premier plan les impressions plutôt que les faits et qui utilisent toutes les ressources de la syntaxe japonaise. Les Notes de

8. Traduit en français par R. SIEFFERT : Le Conte du Coupeur de bambous, Paris, 1992.

9. Le Roman de l'arbre creux, en 20 volumes, est l'histoire sur plusieurs générations d'une famille aristocratique où se transmet l'art du luth en lien avec les intrigues du palais autour de la succession impériale. Cette œuvre qui, par de nombreux aspects, annonce le Roman du Genji ne peut se comparer à celui-ci du point de vue de la cohérence de l'intrigue et de l'art de l'écriture. Il en existe une traduction légèrement abrégée en anglais : The Tale of the Cavern, trad. ZIRO URAKI, Tôkyô, 1984. Plus court et mieux construit, le Roman de la chambre basse, en quatre volumes, relate le destin, d'abord malheureux, puis heureux, d'une jeune femme d'ascendance royale, persécutée par sa belle-mère puis vengée de ses malheurs par son époux, un jeune aristocrate que sa carrière conduit au sommet du pouvoir : Ochikubo Monogatari or the Tale of the Lady Ochikubo, trad. W. WhiteHouSE, E. YANAGISAWA, Tôkyô, 1965 ; voir aussi S. MAUCLAIRE, Du conte au roman : un «Cendrillon » japonais du $X^{e}$ siècle. L'Ochi-kubo-monogatari, Paris, 1984. 
chevet, suite de notations variées sans ordre ni plan, sont célèbres par les listes d'éléments hétéroclites qu'affectionne Sei Shônagon, mais aussi par l'acuité de l'observation et la liberté de jugement dont elle fait preuve dans la peinture de la société de cour de son temps.

\section{Le Roman du Genji}

Considéré comme un des sommets de la littérature japonaise, le Roman du Genji naît à la confluence de toutes ses influences à un moment qui correspond à l'apogée de la puissance de la cour et des régents Fujiwara, dans l'entourage immédiat du plus puissant d'entre eux, Fujiwara no Michinaga. Constitué de cinquante-quatre livres, il évoque l'enfance puis les aventures amoureuses et la carrière brillante du Genji, prince impérial réduit à l'état de sujet quoiqu'il présente tous les signes d'une vocation royale, qui sera néanmoins conduit par son talent au sommet de la fortune terrestre en parvenant à faire de sa fille unique une impératrice et en devenant ainsi l'aïeul d'un empereur. Cette brillante réussite politique est doublée d'une trame secrète. Fils d'une favorite impériale prématurément disparue, le Genji a été élevé au palais auprès de son père et de la nouvelle favorite de celui-ci, la dame Fujitsubo, dont il s'éprend. De cet amour interdit et malheureux naîtra un enfant qui montera sur le trône et qui participera à la fortune du héros, tout en lui rappelant sa faute. Comme par rétribution, le Genji vieillissant sera à son tour victime d'un adultère, et deviendra le père putatif d'un enfant qui, en réalité, n'est pas le sien. Cependant, autant qu'homme d'État, le Genji est un amateur de galanterie. Il collectionne les aventures amoureuses tout en restant fidèle à l'image de la femme idéale qu'il a trouvée en la personne de l'inaccessible Fujistubo, et qu'il retrouve encore en celle de dame Murasaki, une parente de cette dernière, qu'il enlève encore jeune et élève auprès de lui pour en faire son épouse. Il finit par l'installer dans un quartier de la somptueuse demeure qu'il fait construire sur la Sixième Avenue pour y réunir ses diverses épouses. Comme elle ne lui donne pas d'enfant, il la charge d'élever sa fille, née d'une liaison qu'il a eue pendant son exil et qui deviendra impératrice. La mort du Genji intervient peu après celle de Murasaki, au $41^{\mathrm{e}}$ livre dont le texte n'a pas été écrit et dont il n'existe que le titre : «L'Occultation dans les nuages ». Le roman se prolonge alors dans les livres dits d'Uji, qui relatent les aventures amoureuses, le plus souvent contrariées, du fils putatif du Genji, Kaoru (le Parfumé), ainsi que de son royal petit-fils le prince Niou (l'Embaumant), et les tourments que leur rivalité inflige à trois sœurs installées dans une résidence à l'écart de la capitale sur la rivière Uji. La troisième, Ukifune (Barque flottante), partagée entre les deux jeunes gens, se jette dans la rivière 
puis, sauvée des eaux, décide d'entrer en religion et refuse tout contact avec Kaoru, qui cherche à la rencontrer après avoir retrouvé sa trace.

Ce bref résumé du roman ne donne qu'une faible idée de la multiplicité des intrigues et des personnages, rassemblés dans une trame d'une parfaite maîtrise et d'une cohérence sans commune mesure avec les romans précédents, qui permet à l'auteur d'intégrer dans le temps de l'histoire, marqué par la succession des règnes, la multitude des temporalités individuelles. On distingue généralement trois grandes parties : la carrière triomphale du Genji depuis son enfance jusqu'au sommet du pouvoir et de la prospérité (livres 1 à 33) ; puis son lent déclin, marqué par le mariage malheureux qu'il conclut à des fins politiques avec une princesse, ce qui brise le cœur de Murasaki, et se terminant par la mort de cette dernière suivie de celle du Genji (livres 34 à 41) ; enfin, après trois livres de transition (4244), les dix livres d'Uji (45-54), dont l'atmosphère sombre tranche avec la splendeur et l'élégance qui entourent le Genji, même à son déclin.

Le Roman du Genji se présente ainsi comme une somme romanesque reprenant de nombreux éléments empruntés aux romans précédents et multipliant les variations comme pour épuiser les possibilités du genre, tout en les intégrant dans une trame unique de grande ampleur, s'étendant sur au moins trois générations. De nombreuses allusions faites à l'art romanesque, comme celles du livre «Les Concours de peintures » étudié dans l'article d'Estelle Leggeri-Bauer, ou du livre «Lucioles » dont il est question dans celui de Daniel Struve, montrent la conscience de son art que possédait l'auteur du Roman du Genji. Comme l'a remarqué le critique Shimizu Yoshiko ${ }^{10}$, les livres du Roman du Genji s'organisent systématiquement en scènes à caractère dramatique faisant la part belle aux conversations. Si cette technique d'exposition est déjà présente dans les romans du $\mathrm{X}^{\mathrm{e}}$ siècle, le Roman du Genji s'en distingue par l'intensité dramatique qu'il parvient à conférer aux scènes romanesques et la place qu'il accorde au monde intérieur des personnages. Ce dernier est notamment rendu au moyen des nombreux poèmes ou citations poétiques insérés dans le récit, mais aussi grâce au recours systématique à des éléments empruntés à la nature. La poésie japonaise waka est au centre de l'univers romanesque du Roman du Genji, comme le montre l'article de Sumie Terada, mais les allusions à la poésie chinoise, notamment à celle de Bai Juyi, sont également nombreuses ${ }^{11}$. Cette présence de l'intériorité des personnages est rendue possible par un dispositif

10. Y. SHIMIZU, «Bamen to jikan» (La scène et le temps), dans Genji monogatari no buntai to hôhô (Le Roman du Genji, son style et sa méthode d'écriture), Tôkyô, 1980, repris dans Shimizu Yoshiko ronbun shû (Recueil d'articles de Shimizu Yoshiko), vol. I, Tôkyô, 2014, p. 191.

11. K. Hinata éd, Genji monogatari to kanshi no sekai : Hakushi monjyû o chûshin ni (Le Roman du Genji et l'univers de la poésie chinoise : autour du Recueil de BAI Juyi), Tôkyô, 2009. 
narratif qui permet au narrateur de s'effacer presque entièrement derrière les voix des personnages, de façon à pouvoir faire entendre directement leurs discours ou leurs pensées et rendre au plus près leurs intonations. Le narrateur intervient parfois pour apporter son commentaire, mais sans jamais décliner son identité ou apparaître sous les traits d'un personnage. Sa manière de parler ou l'usage qu'il fait des formules de politesse permettent de le situer à la place d'une de ces nombreuses dames de compagnies qui se pressaient dans les maisons des grands dignitaires et qui commentaient volontiers les faits et gestes de leur maîtres, ou encore qui leur contaient pour les divertir des histoires en s'aidant d'illustrations. Parmi les autres traits distinctifs du Roman du Genji, Shimizu Yoshihiko souligne encore la vigueur de la construction des phrases, permettant à l'auteur de rendre dans toute leur complexité les relations entre les personnages, une maîtrise stylistique qu'elle attribue à sa vaste culture littéraire et à sa connaissance des lettres chinoises.

En effet, si, contrairement au poèmes, les romans de l'époque de Heian n'étaient jamais signés, la tradition et les témoignages concordent pour attribuer les Roman du Genji à un auteur bien déterminé, une femme issue de la noblesse intermédiaire et connue sous le nom de Murasaki Shikibu, nom d'usage dont le premier élément pourrait être emprunté à celui de son personnage (la dame Murasaki), tandis que le second désigne le Département des rites, où son père avait eu un poste. Née aux environs de 973, Murasaki Shikibu aurait perdu sa mère dès son plus jeune âge, puis aurait été élevée par son père Fujiwara no Tametoki (945 ?-après 1018), un des meilleurs lettrés de son temps, qu'elle aurait suivi aux environ de ses vingt-cinq ans à son poste de gouverneur de la riche province d'Echizen, obtenu après une longue période de disgrâce. Elle aurait ensuite été mariée à un ami de son père, de vingt ans son aîné, qu'elle perdit bientôt, et c'est ainsi qu'elle serait entrée quelques années plus tard au service du palais auprès de l'impératrice Shôshi, fille de Michinaga. Nous ignorons la date du début de la rédaction du Roman du Genji, mais des indications incluses par Murasaki Shikibu dans son journal permettent de supposer que deux ou trois ans après son entrée au palais, soit vers 1008 , la rédaction du roman était déjà assez avancée ${ }^{12}$. On estime qu'elle serait morte en 1014. Dans ses notes journalières, elle rapporte le compliment que lui a adressé l'empereur Ichijô déclarant : «Cette femme a dû lire les Chroniques du Japon! Elle me paraît posséder un grand savoir, en vérité ${ }^{13}$ ! » Ainsi, la qualité d'auteur du Roman du Genji de Murasaki Shikibu est attestée par l'auteur elle-même,

12. Y. SHIMIZU, Murasaki Shikibu, coll. Iwanami shinsho, Tôkyô, 1973, p. 152-153. S'appuyant sur la lecture du journal de Murasaki Shikibu, Shimizu estime que le livre XIX, « À corps perdu », aurait déjà été écrit à cette date.

13. Murasaki ShIKIBU, Journal, trad. R. SiEFFERT, Paris, 1978, p. 72. 
quand bien même on ne saurait affirmer avec une certitude absolue qu'elle en a rédigé elle-même tous les livres. Cependant, un témoignage ancien, celui de la narratrice et de l'auteur des Mémoires de Sarashina (écrit vers 1059), qui relate les longs efforts qu'elle fit pour réunir les cinquante-quatre livres du Roman du Genji, à son retour à Heian-kyô en $1021{ }^{14}$, laisse penser que le roman existait bel et bien sous une forme voisine de celle que nous connaissons à une date proche de sa rédaction, quelques années seulement après la mort de Murasaki Shikibu.

Ces témoignages, dont le plus ancien remonte au temps même de son écriture, attestent de la réception immédiate de l'œuvre de Murasaki Shikibu et de la reconnaissance de son importance dès sa première diffusion. En l'absence d'histoire officielle, dont la compilation avait été abandonnée par la cour au début du X $\mathrm{X}^{\mathrm{e}}$ siècle, le Roman du Genji pouvait servir de monument à la splendeur de la cour à l'époque de Fujiwara no Michinaga, tout en proposant une profonde leçon morale et politique, à l'instar de la tradition historique chinoise remontant au chef-d'œuvre de Sima Qian ${ }^{15}$. L'affaiblissement ultérieur du pouvoir des régents Fujiwara, puis de la cour elle-même, n'allait pas compromettre la fortune du roman, dont le statut d'œuvre classique et de témoin de la grande époque ne sera plus guère contesté. Le Roman $d u$ Genji, lu et commenté dans les écoles poétiques de waka, continua à nourrir la culture nationale. La poésie en chaîne médiévale, héritière du waka, en fit également une œuvre canonique, et des listes de mots du Roman $d u$ Genji et de ses principaux poèmes furent constituées à l'usage des pratiquants de cet art.

Si l'accès au texte du Roman du Genji resta réservé aux seuls membres des familles aristocratiques ou des écoles poétiques jusqu'à la diffusion du roman sous forme imprimée au XVII siècle, certains de ces motifs seront popularisés dès le « Moyen Âge » par le théâtre nô et d'autres genres populaires liés à la musique et à la danse, comme le sôka ou le kôwakamai ${ }^{16}$. Enfin, on ne saurait sous-estimer l'influence que le Roman du Genji a exercée sur la peinture, dont on peut se faire une idée très complète

14. Le Journal de Sarashina, trad. R. SIEFFERT, Paris, 1978, p. 43.

15. T. TANIGUCHI, Yomitsugareru Shiki : Shiba Sen no denki bungaku (La réception des Mémoires historiques : la littérature biographique de Sima Quian), Tôkyô, 2012,p. 81-91.

16. Le sôka (littéralement « chant rapide ») est un poème destiné à être chanté, constitué à partir de citations de classiques chinois ou japonais, qui fut en vogue parmi les guerriers dans la seconde moitié du XIII ${ }^{\mathrm{e}}$ siècle. Voir N. TONOMURA, « Le récit d'itinéraire dans le sôka et les arts de la scène du Japon médiéval », dans C. SAKAI et al. dir., Les Rameaux noués, Paris, 2013. Le kô-wakamai, lui, est un genre narratif, également en vogue parmi les guerriers des $\mathrm{XIV}^{\mathrm{e}}$ et $\mathrm{XVI}^{\mathrm{e}}$ siècles, dont la récitation s'accompagnait de danse. Il joua un rôle important dans la diffusion de l'héritage classique au sein de la société guerrière du Moyen Âge tardif. 
en consultant l'édition illustrée due à Estelle Leggeri-Bauer publiée en 2007 par les éditions Diane de Selliers ${ }^{17}$.

À l'époque moderne (XVII ${ }^{\mathrm{e}}$-XIX ${ }^{\mathrm{e}}$ siècles), l'éclatement des écoles poétiques et le développement de l'imprimerie élargirent encore le cercle des lecteurs. Le Roman du Genji était certes toujours lu par les poètes, mais aussi par les spécialistes des études japonaises et même par les penseurs confucéens, fascinés par la description des rites de la cour. Plus généralement, un large public cultivé accède alors à une connaissance du roman, soit directe, soit par le truchement de versions abrégées, adaptées ou de données fournies par divers manuels et encyclopédies. Au XVIII ${ }^{\mathrm{e}}$ siècle, le mouvement des Études nationales - et en particulier le penseur Motoori Norinaga - voit dans le Roman du Genji un témoignage unique de la sensibilité proprement japonaise que résume le concept de mono no aware («l'émouvante intimité des choses »), et cette relecture continue à influer sur la perception actuelle de cette œuvre, même si la recherche la plus récente propose des approches toujours plus diversifiées ${ }^{18}$. L'article d'Anne Bayard-Sakai fait le point sur la réception du Roman du Genji dans les milieux littéraires du Japon contemporain et démontre à quel point le roman lui-même et la société aristocratique dont il dresse le tableau continuent à servir de références pour la culture japonaise, en même temps qu'il s'affirme comme une œuvre incontournable de la littérature mondiale.

Daniel Struve - Université Paris-Diderot/Centre de recherche sur les civilisations de l'Asie orientale (CRCAO)

Terada Sumie - Inalco/Centre d'Études Japonaises (CEJ)

\section{Note sur la prononciation}

La transcription des mots japonais est fondée sur le sytème Hepburn.

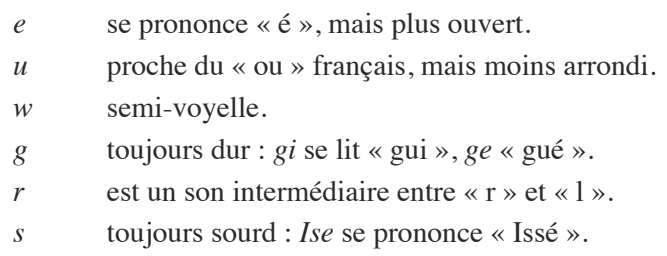

17. MurAsAKI ShIKIBU, Le Dit du Genji illustré par la peinture traditionnelle japonaise, trad. R. SIEFFERT, commentaires iconographiques E. LEGGERI-BAUER, Paris, 2007.

18. J. Pigeot, «Le système de lecture de Motoori Norinaga (1730-1801) », dans F. GIRARD et al. éd., Repenser l'ordre, repenser l'héritage : paysage intellectuel du Japon (XVII ${ }^{e}$-XIX $X^{e}$ siècles), Genève, 2002. Sur la réception du Roman du Genji, cf. T. HARPER et al. éd., Reading The Tale of Genji, New York, 2015, où sont traduits de nombreux textes critiques sur le Roman du Genji datant de toutes les époques. 
sh $\quad$ est une fricative proche du « $\mathrm{ch} »$ français.

ch est une affriquée proche de «tch ».

$j \quad$ est l'équivalent sonore de $s h$ ou de $c h$ proche de $« \mathrm{j} »$ ou $« \mathrm{dj} »$.

Le point marque la dissociation entre un $n$ et la syllabe qui le suit : Man.yôshû. Le $n$ note une consonne nasale et non pas comme en français la nasalisation de la voyelle précédente : Genji se prononce « Guenne-dji ».

L'accent circonflexe indique qu'une voyelle est longue.

\section{Bibliographie sélective}

\section{Ouvrages généraux}

Dictionnaire historique du Japon, éd. IWAO S. et al., Paris, 2002 (2 vol.) (1 ${ }^{\text {re }}$ publication : Tôkyô, 1963-1995).

Dictionnaire des sources du Japon classique, éd. J. PigGotT, I. Smits, I. VAN PUT, M. VieILlard-BARON, C. VON VERSCHUER, Paris, 2006.

\section{Histoire et culture du Japon ancien}

BRISSET C. A., À la croisée du texte et de l'image : paysages cryptiques et poèmes cachés (Ashide) dans le Japon classique et médiéval, Paris, 2009.

FRANK B., Amour, colère, couleur : essais sur le bouddhisme au Japon, Paris, 2000.

HérAIL F., La Cour du Japon à l'époque de Heian, aux Xe et XI siècles, Paris, 1995.

Herail F., Esmein J., Macé F., NinOmiYa H., SouYri P., Histoire du Japon, Lyon, 1990 (nouvelle édition : Paris, 2009).

ÔTsu T., Nihon no rekishi, vol. 6, Michinaga to kyûtei shakai, « Kôdansha gakujutsu bunko », Tôkyô, 2009.

ROBERT J.-N., Les Doctrines de l'école japonaise Tendai au début du IXe siècle, Gishin et le Hokke-shû gi shû, Paris, 1990.

SOUYRI P. F., Nouvelle Histoire du Japon, Paris, Perrin, 2010.

UEDa N., Nihon no rekishi, vol. 5, Ôchô no kizoku, « Chûkô bunko », Tôkyô, 1973.

VON VERSCHUER C., Le Riz dans la culture de Heian. Mythe et réalité, Paris, 2003.

YAMANAKA H. et SUZUKI K. éd., Heian kizoku no kankyô : Heian jidai no bungaku to seikatsu, Tôkyô, 1994.

\section{Littérature japonaise de l'époque de Heian}

Cipango Cahiers d'études japonaises, numéro hors série, Autour du Genji monogatari, dir. TERADA S., 2008. [ En ligne] https://cipango.revues.org/601

Cipango French Journal of Japanese studies, English Selection, On the Tale of Genji : Narrative, Poetics, Historical Context, dir. FuJIWARA D., 3 (2014) (avec un choix d'articles différent de la version française).

KATAGIRI Y., Tsunoda B.éd., Genji monogatari to Murasaki Shikibu : kenkyû no kiseki, « Kadokawa Gakujutsu shuppan », Tôkyô, 2008 (2 vol.).

Konishi J., A History of Japanese Literature, trad. A. GATTEN, New York, 1984-1991 (3 vol.).

Pigeot J., L'Âge d'or de la prose féminine au Japon (Xe-XI siècles), Paris, 2017.

Pigeot J., Questions de poétique japonaise, Paris, 1997.

SHIRANE H., The Bridge of Dreams.A poetics of « The Tale of Genji », Stanford, 1987.

Struve D., Tschudin J.-J., La Littérature japonaise, Paris, « Que sais-je ? », 2016.

SHIMIZU Y., Shimizu Yoshiko ronbunshû, Tôkyô, 2014 (3 vol.). 
SHIMIZU Y. et al., Genji monogatari tekagami, Tôkyô, 1975.

TERADA S., Figures poétiques japonaises. La genèse de la poésie en chaîne, Paris, 2004.

\section{CEuvres de l'époque de Heian traduites en langues occidentales}

AKAZOME EMON (la première partie attribuée à), A Tale of Flowering Fortunes (Eiga monogatari), trad. W. MCCullough et H. C. MCCullough, Stanford, 1980.

ANONYME, Le Conte du Coupeur de bambous, trad. R. SIEFFERT, Paris, 1992.

ANONYME, Contes d'Ise, trad. G. RENONDEAU, Paris, 1969.

ANONYME, Contes de Yamato, trad. R. SIEFFERT, Paris, 1979.

AnONYMes, De la création des jardins. Traduction du Sakutei-ki, trad. M. VIEILLARD-BARON, Paris, 1997.

ANONYME, Histoires qui sont maintenant du passé, trad. B. FRANK, Paris, 1968.

ANONYME, Okagami, The Great Mirror: Fujiwara Michinaga (966-1027) and His Times. A study and Translation, trad. H. C. McCullough, Princeton, 1980.

FUJIWARA no Kintô (compilé par), Recueil des Joyaux d'or et d'autres poèmes, trad. M. VIEILLARD-BARON, Paris, 2015.

FuJIWARA no Kintô (compilé par), Wa-kan Rôeishû, trad. J. T. RIMER, J. CHAVES, New York, 1997.

FuJIWARA no Michinaga, Notes journalières de Fujiwara no Michinaga, ministre à la Cour de Hei.An (995-1018), trad. F. HÉRAIL, Genève-Paris, 1987-1991 (3 vol.).

IZUMI SHIKIBU, Izumi-Shikibu, Journal et poèmes, trad. R. SIEFFERT, Paris, 1989.

KI no Tsurayuki et al. (compilé par), Le Monument poétique de Heian : le Kokinshû, trad. G. BONNEAU, Paris, 1933-1935 (3 vol.).

Mémoires d'une Éphémère (954-974) par la mère de Fujiwara no Michitsuna, trad. J. PIGEOT, Paris, 2006.

MURASAKI SHIKIBU, Journal, trad. R. SIEFFERT, Paris, 1978.

MurAsaki Shikibu, Le Dit du Genji, trad. R. SiefFERT, Paris, 1988 (2 vol.) (rééd. Lagrasse, 2011).

MURASAKI SHIKIBU, Le Dit du Genji illustré par la peinture traditionnelle japonaise, trad. R. Sieffert, commentaires iconographiques E. Leggeri-Bauer, Paris, 2007.

SEI ShÔnagon, Notes de chevet, trad. A. BEAUJARD, Paris, 1985.

SugAwARA no Takasue no musume (Fille de Sugawara no Takasue), Le Journal de Sarashina, trad. René Sieffert, Paris, 1978. 
